\title{
DWEDDEEC: Distance Aware Waiting based EDDEEC Protocol for Hetrogeneous WSNS
}

\author{
Rajeev Kumar \\ Assistant Professor \\ DAV Institute of Engineering \& \\ Technology, Jalandhar, \\ Punjab, India
}

\author{
Rajdeep Kaur \\ DAV Institute of Engineering \& \\ Technology, Jalandhar, Punjab, \\ India
}

\author{
Reeta Bhardwaj \\ DAV Institute of Engineering \& \\ Technology, Jalandhar, Punjab, \\ India
}

\begin{abstract}
As Wireless Sensor Networks (WSNs) contain numerous sensor nodes containing limited battery power that collect and transmit the data collected from all the nodes towards the Base Station which needs much energy utilization. There are several routing protocols which are already proposed under this consideration attaining effectiveness of energy in heterogeneous circumstances. Although, every protocol is unsuitable for heterogeneous WSNs. Efficiency of WSNs declines as varying the heterogeneity of sensor nodes. Though EDDEEC has depicted quite considerable outcomes compared to already developed existing WSNs protocols but it has ignore the utilization of waiting time of node to become cluster heads (CHs). Therefore might a few nodes will not turn out to be $\mathrm{CHs}$ for a very long time still they contain more confidence to become CHs. Therefore to conquer this issue we have used a waiting counter called $\mathrm{W}_{\mathrm{r}}(\mathrm{i}) . \mathrm{W}_{\mathrm{r}}(\mathrm{i})$ is then total number of waiting rounds for a given node to become $\mathrm{CH}$. It will generate a +ve value that will constantly amplify the node to $\mathrm{T}(\mathrm{n})$ value for a specified node to become $\mathrm{CH}$. So it will produce more optimized CHs. For getting the better results more we have used the minimum allowed distance $(\mathrm{MDCH})$ between two $\mathrm{CHs}$ to cover the sensor field in the most proficient manner.
\end{abstract}

\section{General Terms}

Energy efficiency, Throughput, network Lifetime.

\section{Keywords}

WSNs, DEEC, heterogeneity, super nodes, T-absolute

\section{INTRODUCTION}

Wireless sensor networks are a vast field of sensor nodes. WSNs involve of tiny nodes containing detecting, calculation, and wireless interaction and transmission potential. There is a variety of routing, data broadcasting, and power management protocols are exclusively planned for WSNs where energy alertness is a crucial design apprehension. Wireless communication mechanization keeps on growing in various regions to give new prospects for communications and facilities. This is exciting part is WSN. With the development in micro-electro mechanical machines, sensor machines can be designed as little as lightweight wireless nodes. WSN are extremely dispersed networks of these types of sensor nodes, and their deployment in great numbers to observe the atmosphere or manufacture systems. There is an increasing requirement for the nodes to tackle extra difficult tasks in data acquirement and processing, and energy saving results remains a main need for such power based batteries sensor nodes. Three major functions are performed by three sensor subsystems the surroundings sensor; the data processor that performs local calculations on the data sensed; and the communicator that performs information swap between neighboring nodes [1]. Every sensor node is generally controlled in their energy values, capability of operating power and sensing. Though, networks of such sensors provide grow to a network that is robust, correct, and reliable network. The sensors can work together and help with each other, choose heads collect their data and then broadcast a more results from the sensing [2]. A lot of learning on WSN has been carried out in Labs. WSN technology is progressively more requiring by latest applications in a variety of areas, WSN are made up of thousands of small sensor nodes that cover a huge geographical area. These sensors are capable to communicate with each other to mutually sense things, gather data and broadcast information. Though, as sensors are generally little in dimensions, they have numerous physical constraints - like battery, processing power and storage. Due to those restrictions, energy-efficient methods are major research challenges in WSN There are number of protocols for heterogeneous and homogeneous WSN environment. As in homogeneous environment Low-Energy Adaptive Clustering Hierarchy (LEACH) [5], Hybrid Energy-Efficient Distributed clustering (HEED) [8], Power Efficient Gathering in Sensor Information Systems (PEGASIS) [7], these are some algorithms that are created for only one type of nodes. The energy level of these nodes is equal. Whereas, for heterogeneous WSN environment the protocols like Stable Election Protocol (SEP) [9], Distributed Energy-Efficient Clustering (DEEC) [10], Developed DEEC (DDEEC) [11], Enhanced DEEC (EDEEC) [12] and Threshold DEEC (TDEEC) [13] are created.

This paper described various heterogeneous protocols. The energy levels of these protocols are represented in two level, three levels and multilevel heterogeneity level. The study and analysis of the performance of protocols EDEEC, DDEEC, EDDEEC and the proposed protocol DWEDDEEC is covered in this paper. In the heterogeneous WSNs environment there are normal nodes, intermediate nodes, advanced nodes and super nodes. Every protocol concentrates only to achieve better stability time, extended network lifetime and good throughput. All the nodes in network contains same proportion of normal, advanced and super nodes alongside the multilevel heterogeneous WSNs. This has been discovered that every protocol that is different among all the other protocols in terms of stable periods, network lifetime. The protocols EDEEC and EDDEEC achieve great network lifetime performance when they are in three level heterogeneous WSNs containing much energy level distinction between normal, advanced and super nodes 
regarding stability period. However, it is deficient in results as contrast with DDEEC.

The rest of the paper is ordered as follows: section 2 briefly reviews the related work. In section 3 , describes the network model. In section 4, radio dissipation model is explained. Proposed overview of Heterogeneous Protocols explained in section 5.In section 6, elaborates the performance of different DEEC protocols by simulations and comparison. At last, section 7 provides the conclusion and future work.

\section{RELATED WORK}

W.R. Heinzelman, et al. [5] proposed a LEACH clustering algorithm for homogeneous WSNs in which nodes randomly choose among them to be $\mathrm{CHs}$ and transmit this choice criterion over the whole network to issue energy load. G. Smaragdakis, et al. [9] introduced SEP protocol SEP is relied on weighted election possibilities of every sensor node to turn out to be $\mathrm{CH}$ as to the residual energy in every node Heterogeneity-aware Hierarchical Stable Election Protocol (HSEP) [16], protocol is heterogeneous-aware in the fact that the initial energy of a node comparative to the other nodes in the network is relied on the weighted election probabilities.

Extended Stable Election Protocol (ESEP) [17] three-tier clustered heterogeneous network is defined where the intermediate and advanced nodes choose as $\mathrm{CHs}$ for the growing quantity of rounds relied on their advanced initial energy comparative to other sensor nodes. The $\mathrm{CH}$ selection procedure is regarded relied on the battery power and remaining energy of the sensor node

L .Qing, Q. Zhu and M. Wang [10] the election of CHs is done from a possibility lied on the proportion among remaining energy of every node and the average remaining energy of the sensor network. The numbers of rounds for being $\mathrm{CHs}$ for sensor nodes are dissimilar according to the sensor node's initial and remaining energy. Brahim Elbhiri, et $a l$. [11] introduced a protocol DDEEC; the protocol is DEEC relied and perform same function in which all the sensor nodes utilize initial energy and residual energy level for election of CHs. To enhance the DEEC protocol outcomes, the DDEEC executed a stable and dynamic method to allocate the exhausted energy better rightfully among sensor nodes.

P. Saini et al. [12] proposed a protocol EDEEC technique is relied on DEEC with accumulation of super nodes. They have comprehensive the DEEC upto three-level heterogeneity in sensor nodes. $\mathrm{CH}$ algorithm is broken down into rounds. At every round sensor node make a decision whether to be a $\mathrm{CH}$ relied on threshold computed by the recommended proportion of $\mathrm{CHs}$ for the sensor network and the amount of periods the sensor node has been a CH far away. Parul Saini and Ajay K Sharma [13] proposed a protocol TDEEC On every round, sensor nodes make a decision whether to be a $\mathrm{CH}$ or not with deciding a likely random number among 0 and 1 . If value is smaller than threshold value (Ts) then sensor node make a choice to be a CH for the present round. M.Y. Khan et al. [14] developed a protocol called H-DEEC, this protocol is founded on DEEC method for heterogeneous WSNs, where all the sensor nodes make use of initial and remaining energy level for $\mathrm{CH}$ selection. Every sensor node in the network contain the data of the whole the other associate sensor nodes.

N. Javaid et al. [15] developed EDDEEC protocol exercises the idea of possibilities for $\mathrm{CH}$ election founded on initial, residual energy level of the nodes and average remaining energy of the sensor network.

\section{HETROGENEOUS WSN MODEL}

Here in this segment there are $\mathrm{N}$ number of nodes scattered in a square area of dimension $\mathrm{M} \times \mathrm{M}$. The WSN which is heterogeneous WSNs contain two, three or multi types of nodes that are equipped with different proportion of energy which is called one level, two level and multilevel heterogeneous WSNs.

\subsection{Two level Heterogeneous WSN Model}

The networks those are two level heterogeneous WSNs are equipped with two power stages of sensor nodes, regular and innovative nodes. Where $S o$ is the stage of power of regular node and $S o(1+d)$ is the power of advanced nodes containing a periods greater power as in comparison to normal nodes.

Given the count of nodes is $R$ so the variety of innovative nodes is $R m$ where $m$ is the portion of innovative nodes and the variety of regular nodes is $R(1-m)$. So, the complete power of the whole system is given by:

$$
S_{T}=R(1-m) S+R m(1+d) S_{o}=R S_{o}(1+d m)
$$

Therefore it is worth to say that the two level heterogeneous networks contain $\alpha m$ times much battery power compared to homogeneous WSNs.

\subsection{Three level Heterogeneous WSN Model}

In these kinds of networks there are three energy levels of sensor nodes, normal, advanced and super nodes. The energy level of normal node is $S o$, the energy level of every advanced node of fraction $m$ with $d$ times more energy than normal nodes is $S_{o}(1+d)$ and the energy level of every super node of fraction $m_{o}$ with $b$ times greater power compared to the normal nodes is $S_{o}(1+k)$. As $R$ is the total number of nodes in the network, then Rmmo is sum of number of super nodes and $R m(1-m o)$ is the total number of super nodes and $R m(1-$ $m o)$ is the total number of advanced nodes. Here the sum of energy of whole network is specified by:

$$
\begin{gathered}
S_{T}=R(1-m) S_{o}+R m\left(1-m_{o}\right)(1+d) S_{o} \\
+R m_{o} S_{o}(1+k) \\
S_{T}=R S_{o}\left(1+m\left(d+m_{o} k\right)\right)
\end{gathered}
$$

Thus, the three level heterogeneous networks have $\left(d+m_{o} k\right)$ times greater energy as contrast to homogeneous WSNs.

\subsection{Multilevel Heterogeneous WSN Model}

In the environment where multilevel of energy in nodes is equipped called heterogeneous networks, they contain the three levels of energies in sensor nodes. The sensor nodes are equipped with initial energy that is circulated among the set $\left[S_{o} . S_{o}\left(1+d_{m}\right)\right]$, where the minimum energy level value is denoted by $S_{o}$ and the maximum energy value is $d_{m}$. Initially, sensor node $u_{i}$ is prepared by energy of $S_{o}\left(1+d_{i}\right)$, which is $d_{i}$ more compared to than $S_{o}$. Therefore, the complete power of the whole network is specified by:

$$
S_{T}=\sum_{i=1}^{R} S_{o}(1+d)=S_{o}\left(R+\sum_{i=1}^{R} d_{i}\right)
$$

$\mathrm{CH}$ nodes used more power as in comparison to group participant nodes. Therefore after some units stage of power of every node will be dissimilar among every other node. So, the systems having heterogeneity are essential than homogeneous systems. 


\section{RADIO DISSIPATION MODEL}

The energy consumed by network should be as minimum as possible, therefore the radio dissipation model is we use should be appropriate. Here model that shows that 1 bit message is transmitted over a distance $d$ as in [5.6], energy exhausted is then given by:

$$
E_{T x}(1, d)=\left\{\begin{array}{c}
l E_{\text {elec }}+l \varepsilon f_{s} d^{2}, \quad d<d_{o} \\
l E_{\text {elec }}+l \varepsilon m p d^{4}, \quad d \geq d_{o}
\end{array}\right.
$$

Where, Eelec is the energy dispersed for every bit to drive the transmission circuit. $d$ is the separation among transmitter and receiver. But if the distance is minimum than the threshold value, then the model called free space (fs) is utilized otherwise the model called multi path (mp) is utilized. Hence, total energy dissipated throughout the network round is specified by $[5,6]$

$$
E_{\text {round }}=L\left(2 N E_{\text {elec }}+N E_{D A}+k \varepsilon_{m p} d_{\text {toBS }}^{4}+N \varepsilon_{f a} d_{\text {toCH }}^{2}\right.
$$

Here, $K=$ total number of clusters

$E D A=$ Data aggregation cost expensed in $\mathrm{CH}$

dto $B S=$ Average distance among the $\mathrm{CH}$ and $\mathrm{BS}$

dto $\mathrm{CH}=$ Average distance among the cluster members and the $\mathrm{CH}$

$d_{t o C H}=\frac{M}{\sqrt{2 \pi}}, d_{t o B S}=0.765 \frac{M}{2}$

$k_{\text {opt }}=\frac{\sqrt{N}}{\sqrt{2 \pi}} \sqrt{\frac{\varepsilon_{f s}}{\varepsilon_{m p}}} \frac{M}{d_{t o B S}^{2}}$

\section{OVERVIEW OF DISTRIBUTED HETEROGENOUS PROTOCOLS}

\subsection{DEEC}

DEEC [10] is proposed to concern with nodes of heterogeneous WSNs. For $\mathrm{CH}$ selection, DEEC uses initial and residual energy level of nodes. Let $n_{i}$ denote the number of rounds to be a $\mathrm{CH}$ for node $s_{i}$. poptN is the optimal number of $\mathrm{CHs}$ in the network through each round. $\mathrm{A} \mathrm{CH}$ selection condition in DEEC is formulated on energy level of nodes.

1.Firstly, deploy nodes randomly

2.Normal nodes identify :if $(i \geq(m \times n) \quad / / \mathrm{i}$ is the fraction of node, $m$ is the fraction of advance nodes.

3.N(i). $E=E_{o}$

// $E_{o}=$ Initial energy

4.end if

5.Advance nodes identify: if $(i<(m) \times n)$

6.N(i).E $=E_{o} \times(1+a)$

// $\mathrm{a}$ is extra energy for advance nodes.

7.end if

8. Calculate weighted probabilities used to select $\mathrm{CHs:}$

$$
\begin{gathered}
p_{\text {nrm }}=\frac{\mathrm{P}_{\mathrm{opt}}}{(1+\mathrm{m} * \mathrm{a})}, \text { for normal nodes } \\
p_{a d v}=\frac{\mathrm{P}_{\mathrm{opt}}(1+a)}{(1+\mathrm{m} * \mathrm{a})}, \text { for advance nodes }
\end{gathered}
$$

$/ / p_{\text {opt }}$ is the optimum probability forCH selection

9. Calculate thresholds to select $\mathrm{CHs}$

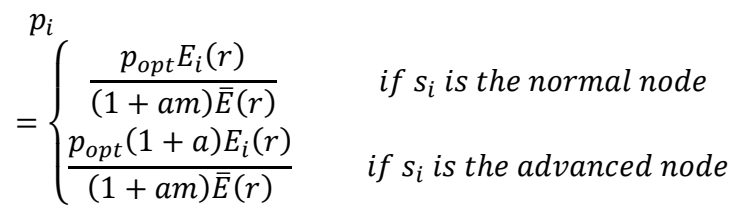

10. Where estimate average energy $E(r)$ of the network for any round $r$.

$$
\bar{E}(r)=\frac{1}{N} E_{\text {total }}\left(1-\frac{r}{R}\right)
$$

$\mathrm{R}$ denotes total rounds of network lifetime and is estimated as follows:

$$
R=\frac{E_{\text {total }}}{E_{\text {round }}}
$$

$E_{\text {total }}$ is total energy of the network where $E_{\text {round }}$ is energy expenditure during each round.

11. Broadcasting of message by elected $\mathrm{CH}$ to sensor nodes (SNs).

12. Allocation of TDMA schedule by $\mathrm{CH}$ to $\mathrm{CMs}$ (Cluster Members).

13. For $\mathrm{CH}$, for $\mathrm{CMs}$

14. Data sent from CMs to CHs as per TDMA schedule.

15. end for $\mathrm{CMs}$

16. Send data from $\mathrm{CHs}$ nodes to BS.

17. end for $\mathrm{CHs}$

18. Evaluate the energy dissipated:

$$
\begin{array}{ll}
\mathrm{E}_{\mathrm{Tx}}(\mathrm{l}, \mathrm{d})=1 \mathrm{E}_{\text {elec }}+1 \varepsilon_{\mathrm{fs}} d^{2}, & \mathrm{~d}<d_{0} \\
\mathrm{E}_{\mathrm{Tx}}(\mathrm{l}, \mathrm{d})=1 \mathrm{E}_{\text {elec }}+1 \varepsilon_{\mathrm{mp}} d^{4}, & \mathrm{~d} \geq d_{0}
\end{array}
$$

19. Repeat steps 12 to 19 until all nodes become dead.

20. End of code

\subsection{DDEEC}

DDEEC utilize the theory for evaluation of average energy in the network and $\mathrm{CH}$ selection algorithm relied on remaining energy as defined in DEEC [10].

1. Firstly, deploy nodes randomly

2. Normal nodes identify :if $(i \geq(m \times n) \quad / / i$ is the fraction of node, $m$ is the fraction of advance node.
3. $N(i) \cdot E=E_{o}$
$/ / E_{o}=$ Initial energy
4. end if
5. Advance nodes identify: if $(i<(m) \times n)$
6. $N(i) \cdot E=E_{o} \times(1+a)$
// $\mathrm{a}$ is extra energy for advance nodes.

7. end if

8. Calculate weighted probabilities used to select $\mathrm{CHs}$ $p_{\text {nrm }}=\frac{\mathrm{P}_{\mathrm{opt}}}{(1+\mathrm{m} * \mathrm{a})}$, for normal nodes 
$p_{a d v}=\frac{\mathrm{P}_{\mathrm{opt}}(1+a)}{(1+\mathrm{m} * \mathrm{a})}$, for advance nodes $\quad / / p_{\text {opt }}$ is the optimum probability for $\mathrm{CH}$ selection

9. Calculate the threshold residual energy level.

$$
T h_{R E V}=E_{o}\left(1+\frac{a E_{d i s N N}}{E_{\text {disNN }}-E_{\text {disAN }}}\right)
$$

$E_{\text {disNN }}$ is the energy dissipated by Normal nodes.

$E_{\text {disAN }}$ is the energy dissipated by advance nodes.

The calculated Threshold residual energy given as:

$$
T h_{R E V}=\left(\frac{7}{10}\right) E_{o}
$$

10. Calculate thresholds to select $\mathrm{CHs}$

$$
p_{i}=\left\{\begin{array}{cc}
\frac{p_{\text {opt }} E_{i}(r)}{(1+a * m) \bar{E}(r)} \quad \text { for Normal nodes, } \\
E_{i}(r)>T h_{R E V} \\
\frac{(1+a) p_{o p t} E_{i}(r)}{(1+a * m) \bar{E}(r)} \quad \text { for Advance nodes, } \\
E_{i}(r)>T h_{R E V} \\
c \frac{(1+a) p_{o p t} E_{i}(r)}{(1+a * m) \bar{E}(r)} \text { for Aadvance, Normal nodes, } \\
E_{i}(r) \leq T h_{R E V}
\end{array}\right.
$$

11. Broadcasting of message by elected $\mathrm{CH}$ to sensor nodes (SNs).

12. Allocation of TDMA schedule by $\mathrm{CH}$ to $\mathrm{CMs}$ (Cluster Members).

13. For $\mathrm{CH}$, for $\mathrm{CMs}$

14. Data sent from CMs to CHs as per TDMA schedule

15. end for CMs

16. Send data from $\mathrm{CHs}$ nodes to BS.

17. end for $\mathrm{CHs}$

18. Evaluate the energy dissipated:

$$
\begin{array}{ll}
\mathrm{E}_{\mathrm{Tx}}(\mathrm{l}, \mathrm{d})=1 \mathrm{E}_{\text {elec }}+1 \varepsilon_{\mathrm{fs}} d^{2}, & d<d_{0} \\
\mathrm{E}_{\mathrm{Tx}}(\mathrm{l}, \mathrm{d})=1 \mathrm{E}_{\text {elec }}+1 \varepsilon_{\mathrm{mp}} d^{4}, & d \geq d_{0}
\end{array}
$$

19. Repeat steps 12 to 19 until all nodes become dead.

\section{End of code}

\subsection{EDEEC}

EDEEC utilize the theory of three level heterogeneous networks as described in [12]. It considers three types of nodes normal, advanced and super nodes relied on initial energy.

1. Firstly, deploy nodes randomly

2. Normal nodes identify : if $(i \geq(m+u) \times n) \quad / / \mathrm{i}$ is the fraction of node, $m$ is the fraction of advance node and $u$ is the fraction of super nodes.

$$
\begin{aligned}
& \text { 3. } N(i) . E=E_{o} \quad / / E_{o}=\text { Initial energy } \\
& \text { 4. end if }
\end{aligned}
$$

5.Intermediate nodes identify: $\quad$ if $(m * n<i<(m+u) \times$ n)
6. $N(i) \cdot E=E_{o} \times(1+b)$

$/ / b$ is extra energy for intermediate nodes

7. end if

8.Advance nodes identify:if $(i<(m) \times n)$

9. $N(i) \cdot E=E_{o} \times(1+a)$

$/ / \mathrm{a}$ is extra energy for advance nodes.

10.end if

11. Calculate thresholds to select CHs

$$
\begin{aligned}
& p_{\text {nrm }}=\frac{\mathrm{P}_{\mathrm{opt}}}{(1+\mathrm{m} * \mathrm{a}+\mathrm{u} * \mathrm{~b})}, \text { for normal nodes } \\
& p_{\text {int }}=\frac{\mathrm{P}_{\mathrm{opt}}(1+b)}{(1+\mathrm{m} * \mathrm{a}+\mathrm{u} * \mathrm{~b})}, \text { for intermediate nodes } \\
& p_{\text {adv }}=\frac{\mathrm{P}_{\mathrm{opt}}(1+a)}{(1+\mathrm{m} * \mathrm{a}+\mathrm{u} * \mathrm{~b})}, \text { for super nodes } \quad / / p_{\text {opt }} \text { is the } \\
& \text { optimum probability for CH selection }
\end{aligned}
$$

$$
p_{i}= \begin{cases}\frac{p_{o p t} E_{i}(r)}{(1+m(a+u * b)) \bar{E}(r)} & \text { if } s_{i} \text { is the normal node } \\ \frac{(1+a) p_{\text {opt }} E_{i}(r)}{(1+m(a+u * b)) \bar{E}(r)} & \text { if } s_{i} \text { is the advance node } \\ \frac{(1+b) p_{o p t} E_{i}(r)}{(1+m(a+u * b)) \bar{E}(r)} & \text { if } s_{i} \text { is the super node }\end{cases}
$$

13. Broadcasting of message by elected $\mathrm{CH}$ to sensor nodes (SNs).

14. Allocation of TDMA schedule by $\mathrm{CH}$ to $\mathrm{CMs}$ (Cluster Members).

\section{For $\mathrm{CH}$, for $\mathrm{CMs}$}

16. Data sent from CMs to CHs as per TDMA schedule

17. end for $\mathrm{CMs}$

18. Send data from $\mathrm{CHs}$ nodes to BS.

19. end for $\mathrm{CHs}$

20. Evaluate the energy dissipated:

$$
\begin{array}{ll}
\mathrm{E}_{\mathrm{Tx}}(\mathrm{l}, \mathrm{d})=1 \mathrm{E}_{\text {elec }}+1 \varepsilon_{\mathrm{fs}} d^{2}, & d<d_{0} \\
\mathrm{E}_{\mathrm{Tx}}(\mathrm{l}, \mathrm{d})=1 \mathrm{E}_{\text {elec }}+1 \varepsilon_{\mathrm{mp}} d^{4}, & d \geq d_{0}
\end{array}
$$

21. Repeat steps 12 to 19 until all nodes become dead

22. End of code

\subsection{EDDEEC}

This paper implements the proposal of possibilities for $\mathrm{CH}$ selection relied on initial, residual energy level of the sensor nodes and average energy of network. The protocol guards the super and advance nodes from over punished, as following a few rounds a few super and advance nodes contain equal remaining energy level same as normal nodes because of constantly $\mathrm{CH}$ selection. EDDEEC computes possibilities of normal, advance and super nodes. These modifications are relied on (ARL) absolute residual energy level $T_{\text {absolute }}$, which is the value in which advance and super nodes contain equal energy level as that of normal nodes. The proposal defines that under $T_{\text {absolute }}$ all normal, advance and super nodes contain equal possibility for $\mathrm{CH}$ selection [15].

1. Firstly, deploy nodes randomly 
2. Normal nodes identify :if $(i \geq(m+u) \times n) \quad / / \mathrm{i}$ is the fraction of node, $\mathrm{m}$ and $\mathrm{u}$ are fractions of advance nodes and intermediate nodes respectively

$$
\begin{aligned}
& \text { 3. } N(i) \cdot E=E_{o} \quad / / E_{o}=\text { Initial energy } \\
& \text { 4. end if }
\end{aligned}
$$

5.Intermediate nodes identify: $\quad$ if $(m * n<i<(m+u) \times$ n)

$$
\text { 6. } N(i) \cdot E=E_{o} \times(1+b) \quad \begin{aligned}
& / / b \text { is extra energy for } \\
& \text { intermediate nodes }
\end{aligned}
$$

7. end if

8. Advance nodes identify:if $(i<(m) \times n)$

9. $N(i) \cdot E=E_{o} \times(1+a) \quad / /$ a is extra energy for

10. end if

11. Calculate weighted probabilities used to select $\mathrm{CHs}$ :

$$
\begin{aligned}
& p_{n r m}=\frac{\mathrm{P}_{\mathrm{opt}}}{(1+\mathrm{m} * \mathrm{a}+\mathrm{u} * \mathrm{~b})}, \text { for normal nodes } \\
& p_{\text {int }}=\frac{\mathrm{P}_{\mathrm{opt}}(1+b)}{(1+\mathrm{m} * \mathrm{a}+\mathrm{u} * \mathrm{~b})}, \text { for intermediate nodes } \\
& p_{a d v}=\frac{\mathrm{P}_{\mathrm{opt}}(1+a)}{(1+\mathrm{m} * \mathrm{a}+\mathrm{u} * \mathrm{~b})}, \text { for advance nodes }
\end{aligned}
$$$$
/ / p_{\text {opt }} \text { is the optimum probability for } \mathrm{CH} \text { selection }
$$

12. Calculate the absolute residual energy level

$$
\begin{gathered}
z=0.7 \\
T_{\text {absolute }}=z * \text { Initilalenergy }
\end{gathered}
$$

Where $\mathrm{z}$ is the fraction to evaluate the absolute residual energy level

13. Calculate thresholds to select $\mathrm{CHs}$

$$
\text { Tnrm }=\frac{P_{n r m} * E_{i}(r) *\left(1+W_{r}(i) * p\right)}{1-p_{n r m}\left(r . \bmod \left(\frac{1}{p_{n r m}}\right)\right)}
$$

for normal nodes, if $\mathrm{E}_{\mathrm{i}}(\mathrm{r})>T_{\text {absolute }}$

$$
\text { Tint }=\frac{\mathrm{P}_{\text {int }} * \mathrm{E}_{\mathrm{i}}(\mathrm{r}) *\left(1+\mathrm{W}_{\mathrm{r}}(i) * p\right)}{1-\mathrm{p}_{\text {int }}\left(\mathrm{r} \cdot \bmod \left(\frac{1}{\mathrm{p}_{\text {int }}}\right)\right)}
$$

for intermediate node if $\mathrm{E}_{\mathrm{i}}(\mathrm{r})>T_{\text {absolute }}$

$$
T a d v=\frac{\mathrm{P}_{\mathrm{adv}} * \mathrm{E}_{\mathrm{i}}(\mathrm{r}) *\left(1+\mathrm{W}_{\mathrm{r}}(i) * p\right)}{1-\mathrm{p}_{\mathrm{adv}}\left(\mathrm{r} \cdot \bmod \left(\frac{1}{\mathrm{p}_{\mathrm{adv}}}\right)\right)}
$$

for advance node if $\mathrm{E}_{\mathrm{i}}(\mathrm{r})>T_{\text {absolute }}$

$$
\text { Tarl }=\frac{\mathrm{P}_{\mathrm{opt}} * \mathrm{E}_{\mathrm{i}}(\mathrm{r}) *\left(1+\mathrm{W}_{\mathrm{r}}(i) * p\right)}{1-\mathrm{P}_{\mathrm{opt}}\left(\mathrm{r} \cdot \bmod \left(\frac{1}{\mathrm{P}_{\mathrm{opt}}}\right)\right)}
$$

\section{for all nodes otherwise}

14. Broadcasting of message by elected $\mathrm{CH}$ to sensor nodes (SNs).
15. Allocation of TDMA schedule by $\mathrm{CH}$ to $\mathrm{CMs}$ (Cluster Members).

16. For $\mathrm{CH}$, for $\mathrm{CMs}$

17. Data sent from CMs to $\mathrm{CHs}$ as per TDMA schedule

18. end for CMs

19. Send data from $\mathrm{CHs}$ nodes to BS.

20. end for $\mathrm{CHs}$

21. Evaluate the energy dissipated:

$$
\begin{aligned}
& E_{T x}(l, d)=l E_{\text {elec }}+l \varepsilon_{f s} d^{2}, \quad d<d_{0} \\
& E_{T x}(l, d)=l E_{\text {elec }}+l \varepsilon_{m p} d^{4}, \quad d \geq d_{0}
\end{aligned}
$$

22. Repeat steps 12 to 19 until all nodes become dead

\section{End of code}

\section{SIMULATIONS AND DISCUSSIONS}

This part defines the simulations and discussions; here we simulate the different heterogeneous clustering protocols in WSN with the help of MATLAB and to make simulations 100 nodes are used randomly deployed in the area of dimension $100 \mathrm{~m} \times 100 \mathrm{~m}$. To make it easy, all the nodes are supposed to be either fixed or micro-mobile and avoid energy loss because of signal collision and meddling among signals of dissimilar nodes that are due to dynamic random channel situations.

Table 1. WSNs Set-up

\begin{tabular}{|l|l|}
\hline Parameter & Value \\
\hline Area(x,y) & 100,100 \\
\hline Base station(x,y) & 50,50 \\
\hline Nodes(n) & 100 \\
\hline Probability(p) & 0.1 \\
\hline Initial Energy & 0.1 \\
\hline transmitter_energy & $50 * 10^{-9}$ \\
\hline receiver_energy & $50 * 10^{-9}$ \\
\hline Free space(amplifier) & $10 * 10^{-13}$ \\
\hline Multipath(amplifier) & $0.0013 * 10^{-13}$ \\
\hline Effective Data aggregation & $5 * 10^{-9}$ \\
\hline Maximum lifetime & 2500 \\
\hline Data packet Size & 4000 \\
\hline m (fraction of advanced nodes) & 0.3 \\
\hline $\begin{array}{l}\text { a (energy factor between } \\
\text { normal and advanced nodes) }\end{array}$ & 3 \\
\hline$m_{o}$ fraction of super nodes & 0.3 \\
\hline
\end{tabular}

\section{PROPOSED ALGORITHM}

1. Firstly, deploy nodes randomly.

2. Normal Nodes Identify, :if $(i \geq(m+u) \times n) \quad / / \mathrm{i}$ is 
the fraction of node, $\mathrm{m}$ and $\mathrm{u}$ are fractions of advance nodes and intermediate nodes respectively.

$$
\text { 3. } N(i) \cdot E=E_{o} \quad / / E_{o}=\text { Initial energy }
$$

4. end if

5. Intermediate nodes identify:if $(m * n<i<(m+$ u) $\times n$ )

6. $N(i) \cdot E=E_{o} \times(1+b)$ $/ / b$ is extra energy for intermediate nodes

7. end if

8. Advance nodes identify:if $(i<(m) \times n)$

9. $N(i) \cdot E=E_{o} \times(1+a) \quad / / \mathrm{a}$ is extra energy for advance nodes.

10. end if

11. Calculate weighted probabilities used to select $\mathrm{CHs:}$

$$
\begin{aligned}
& p_{\text {nrm }}=\frac{\mathrm{P}_{\mathrm{opt}}}{(1+\mathrm{m} * \mathrm{a}+\mathrm{u} * \mathrm{~b})}, \text { for normal nodes } \\
& p_{\text {int }}=\frac{\mathrm{P}_{\mathrm{opt}}(1+b)}{(1+\mathrm{m} * \mathrm{a}+\mathrm{u} * \mathrm{~b})}, \text { for intermediate nodes } \\
& p_{a d v}=\frac{\mathrm{P}_{\mathrm{opt}}(1+a)}{(1+\mathrm{m} * \mathrm{a}+\mathrm{u} * \mathrm{~b})}, \text { for advance nodes } \quad / / p_{\text {opt }}
\end{aligned}
$$

is the optimum probability for $\mathrm{CH}$ selection

12. Calculate the absolute residual energy level

$$
\begin{gathered}
z=0.7 \\
T_{\text {absolute }}=z * \text { Initilalenergy }
\end{gathered}
$$

Where $\mathrm{z}$ is the fraction to evaluate the absolute residual energy level

13. Evaluate the MDCH and waiting nodes

a) $d_{m}(i)$ is either +ve 1 or - ve $1 . \quad / / d_{m}$ is the distance between two $\mathrm{CHs}$

Algorithm is depend upon total $\mathrm{CHs}$ in the network area.

\section{Total Area $=\mathrm{Xm} * \mathrm{Ym}$}

Total Cluster Required $=$ Depend upon probability

Area occupied by one cluster $=$ Total Area/no of Cluster i.e. (Total Area)

$1 / \mathrm{P}$

Now Area Occupied by one cluster $=\underline{(\text { Total Area })}$

$$
\begin{aligned}
& \pi r^{2}=P * \text { Total Area } \\
& r^{2}=(P * \text { Total Area }) * 7 / 22 \\
& r=\sqrt{ }(P * \text { Total Area }) * 7 / 22)
\end{aligned}
$$

Appropriate Distance from One $\mathrm{CH}$ to another $\mathrm{CH}$

$$
=\sqrt{ }(P * \text { Total Area }) * 7 / 22)
$$

So we take this distance is 36 .

$$
d_{m}=1
$$

If $\mathrm{d}>36$ then node can become $\mathrm{CH}$.
End if.

$$
d_{m}=0, \text { if } \mathrm{d}<=36
$$

That node cannot be a cluster head

End if

b) $W_{r}(i)$ is then total number of waiting rounds for a given node to become $\mathrm{CH}$. It must produce a +ve value more than 1 so that it can increase the chance of node to become a CH. $r$ is the current round in WSNs network lifetime, $E_{i}(r)$ is the current energy of each node.

14. Calculate thresholds to select $\mathrm{CHs}$

Tnrm $=\frac{\mathrm{P}_{\text {nrm }} * \mathrm{E}_{\mathrm{i}}(\mathrm{r}) *\left(1+\mathrm{W}_{\mathrm{r}}(i) * p\right) * d_{m}(i)}{1-\mathrm{p}_{\text {nrm }}\left(\operatorname{r.mod}\left(\frac{1}{\mathrm{p}_{\text {nrm }}}\right)\right)}$, for normal nodes, if

$$
\mathrm{E}_{\mathrm{i}}(\mathrm{r})>T_{\text {absolute }}
$$

Tint $=\frac{\mathrm{P}_{\text {int }} * \mathrm{E}_{\mathrm{i}}(\mathrm{r}) *\left(1+\mathrm{W}_{\mathrm{r}}(i) * p\right) * d_{m}(i)}{1-\mathrm{p}_{\text {int }}\left(\operatorname{r.mod}\left(\frac{1}{\mathrm{p}_{\text {int }}}\right)\right)}$, for intermediate nodes,

$$
\text { if } \mathrm{E}_{\mathrm{i}}(\mathrm{r})>T_{\text {absolute }}
$$

$\operatorname{Tad} v=\frac{\mathrm{P}_{\mathrm{nrm}} * \mathrm{E}_{\mathrm{i}}(\mathrm{r}) *\left(1+\mathrm{W}_{\mathrm{r}}(i) * p\right) * d_{m}(i)}{1-\mathrm{p}_{\mathrm{adv}}\left(\operatorname{r.mod}\left(\frac{1}{\mathrm{p}_{\mathrm{adv}}}\right)\right)}$ For advance node if

$$
\mathrm{E}_{\mathrm{i}}(\mathrm{r})>T_{\text {absolute }}
$$

$\operatorname{Tarl}=\frac{\mathrm{P}_{\mathrm{opt}} * \mathrm{E}_{\mathrm{i}}(\mathrm{r}) *\left(1+\mathrm{W}_{\mathrm{r}}(i) * p\right) * d_{m}(i)}{1-\mathrm{P}_{\mathrm{opt}}\left(\operatorname{r.mod}\left(\frac{1}{\mathrm{P}_{\mathrm{opt}}}\right)\right)}$ for all nodes otherwise

15. Broadcasting of message by elected $\mathrm{CH}$ to Sensor Nodes(SNs)

16. Allocation of TDMA schedule by $\mathrm{CH}$ to $\mathrm{CM}$.

17. For $\mathrm{CH}$, for $\mathrm{CMs}$

18. Data sent from $\mathrm{CMs}$ to $\mathrm{CH}$ as per TDMA schedule.

19 end for CMs

20. Sent data from $\mathrm{CHs}$ nodes to BS.

21. end for $\mathrm{CHs}$

22. Evaluate the energy dissipated:

$$
\begin{array}{ll}
\mathrm{E}_{\mathrm{Tx}}(\mathrm{l}, \mathrm{d})=1 \mathrm{E}_{\text {elec }}+l \varepsilon_{\mathrm{fs}} d^{2}, & \mathrm{~d}<d_{0} \\
\mathrm{E}_{\mathrm{Tx}}(\mathrm{l}, \mathrm{d})=1 \mathrm{E}_{\text {elec }}+1 \varepsilon_{\mathrm{mp}} d^{4}, & \mathrm{~d} \geq d_{0}
\end{array}
$$

23. Repeat steps 12 to 22 until all nodes become dead.

24. End of code

\section{PERFORMANCE ANALYSIS}

The existing EDDEEC and the proposed waiting round time and MDCH EDDEEC has been designed and implemented in MATLAB tool. This section contains the comparative analysis between proposed and existing EDDEEC. 


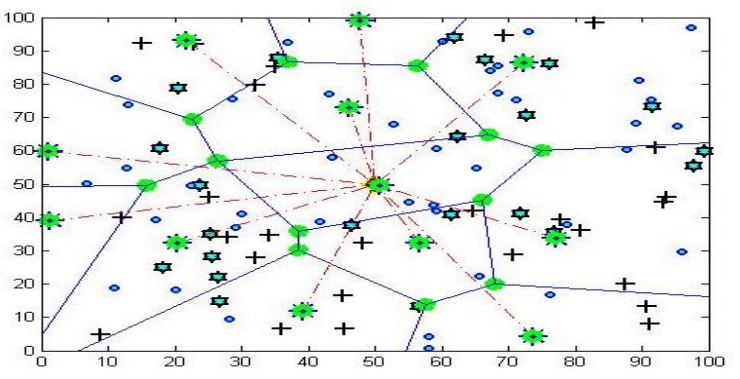

Figure 16 Where Every Sensor Node is Alive

Figure 16 depicts the active environment of the proposed algorithm in which circled nodes are normal nodes, stared nodes are advance nodes, and (+) plus nodes are super nodes. Also diamond is base station of this sensor field.

Figure 17 has shown the some dead nodes with red crossed (X) signs. Dead nodes means it has zero energy.

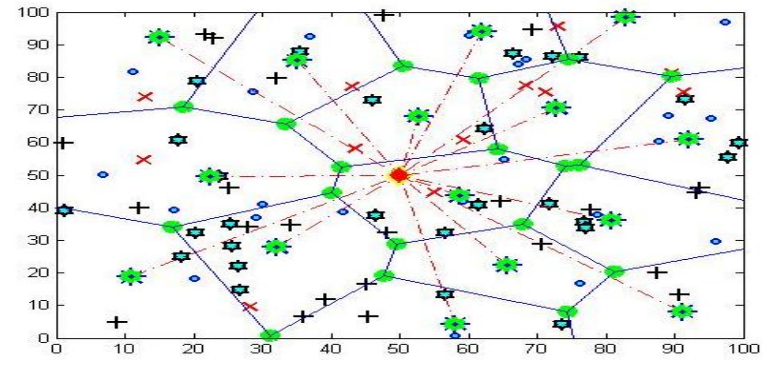

Figure17 where a few sensor nodes become dead

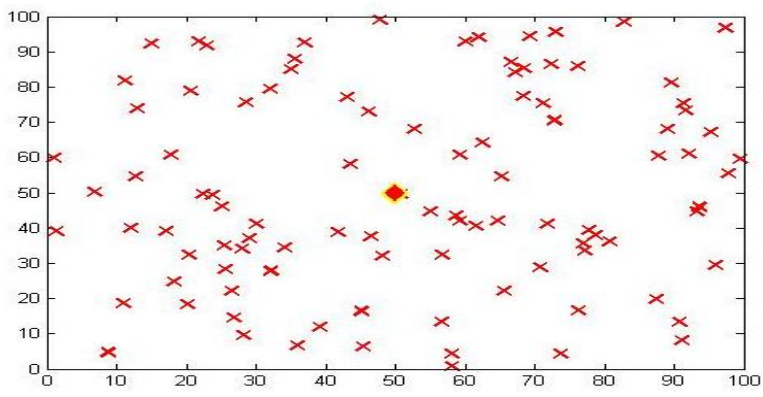

Figure 18 where every sensor node becomes dead

Figure 18 has shown the dead end of sensor field. All nodes are dead. This is also called network lifetime.

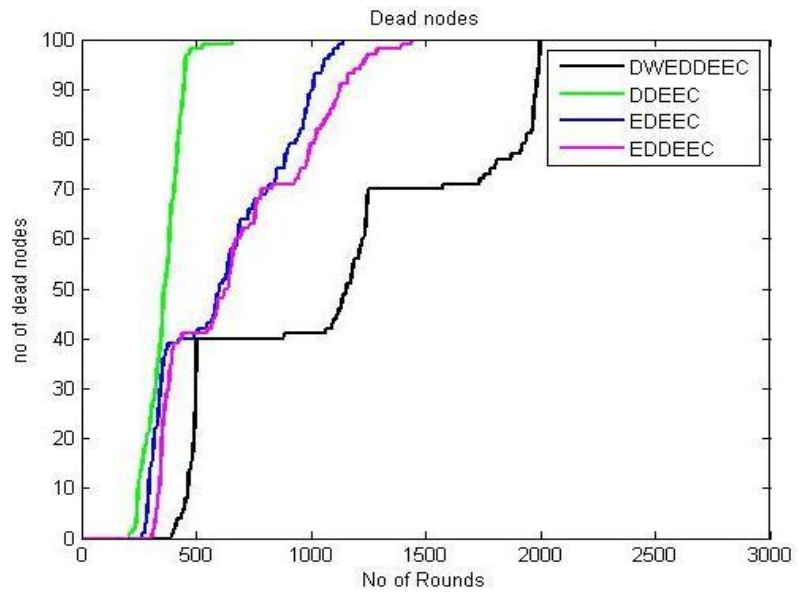

Figure 19 Dead nodes
Figure 19 has shown the analysis of DEAD nodes of DDEEC, EDEEC, EDDEEC and DWEDDEEC. It has been clearly shown that the first node dead time and last node dead time has been prolonged. The first node dead time is 201, 231, 252 and 423 respectively. Last node dead time is $617,1058,1199$ and 1995 respectively. Therefore DWEDDEEC has shown significant improvement over the existing technique.

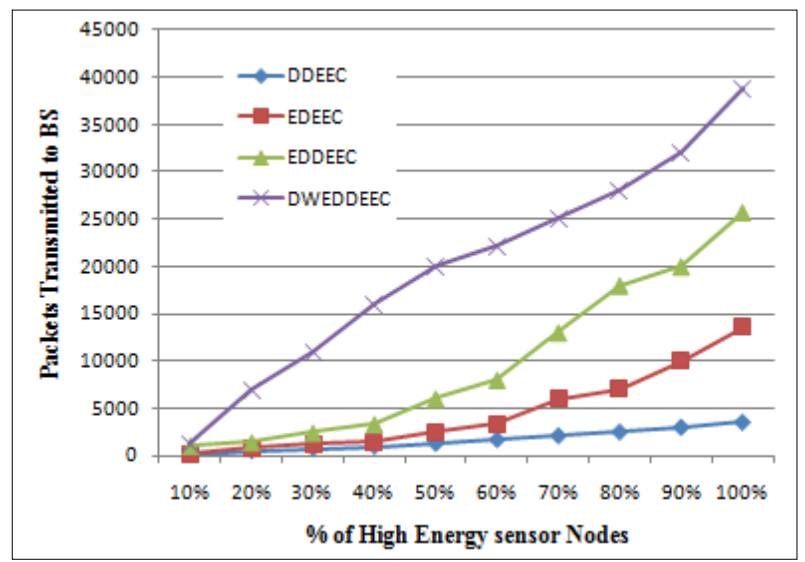

Figure 20 Packets sent to BS

Figure 20 and Figure 21 have shown the packets transmitted to the $\mathrm{BS}$ and $\mathrm{CH}$ respectively.

The comparison of all the protocols that shows the first dead nodes, last dead nodes, tenth dead nodes, packets to BS and packets to $\mathrm{CH}$.

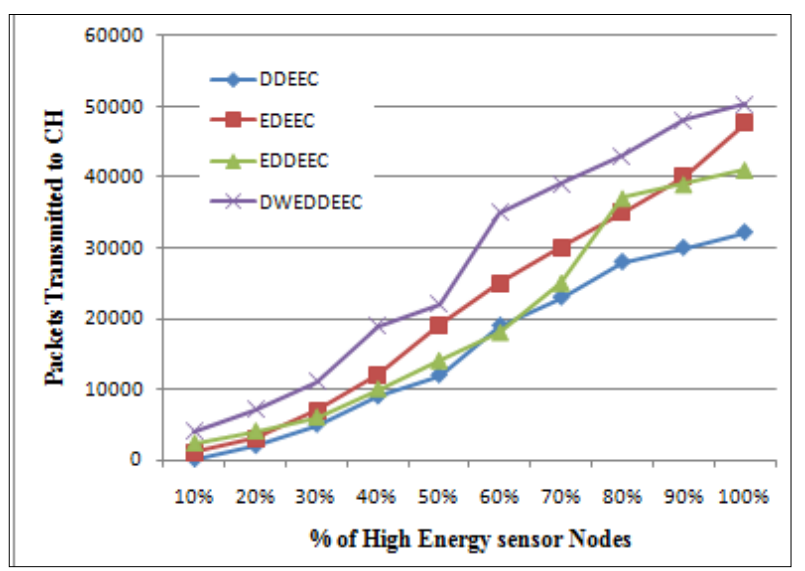

Figure 21 Packets sent to $\mathrm{CH}$

Table 2. Results of Simulation

\begin{tabular}{|l|l|l|l|l|}
\hline Protocols & $\begin{array}{l}\text { First } \\
\text { node } \\
\text { dead }\end{array}$ & $\begin{array}{l}\text { Last } \\
\text { node } \\
\text { dead }\end{array}$ & $\begin{array}{c}\text { Packets } \\
\text { to } \\
\mathrm{CH}\end{array}$ & $\begin{array}{c}\text { Packets } \\
\text { to } \\
\text { BS }\end{array}$ \\
\hline DDEEC & 201 & 617 & 32184 & 3525 \\
\hline EDEEC & 231 & 1058 & 47618 & 13551 \\
\hline EDDEEC & 252 & 1199 & 41033 & 25721 \\
\hline DWEDDEEC & 423 & 1995 & 50331 & 38766 \\
\hline
\end{tabular}




\section{CONCLUSION AND FUTURE WORK}

Though EDDEEC has depicted pretty considerable outcomes over existing WSNs protocols except it has ignore the utilization of waiting time of node to become $\mathrm{CHs}$. Therefore might a few nodes will not turn out to be $\mathrm{CHs}$ for a very long duration still they contain additional confidence to become CHs. Therefore to conquer this, a waiting counter called $W_{r}(i)$ is used. To improve the results further MDCH between two CHs has also been used to deploy the sensor field in the most proficient manner. $\mathrm{MDCH}$ has capability to conquer the issue of the too small and too high $\mathrm{CHs}$. MDCH is based on mathematical radius theory of a circle to evaluate the optimized sensor field distribution. If distance between node going to become $\mathrm{CH}$ and any existing $\mathrm{CH}$ is less than the threshold distance then it will produce a -ve value so node will not become $\mathrm{CH}$. The proposed algorithm is designed and implemented in MATLAB. The comparisons have shown significant improvement over the existing EDDEEC.

In near future we will use ant colony based data aggregation strategy for $\mathrm{CHs}$ to improve the performance of the proposed EDDEEC further.

\section{REFERENCES}

[1] I.F. Akyildiz, W. Su, Y. Sankara subramaniam, E. Cayirci, (2002) "Wireless sensor networks: a survey, Computer Networks", 38 (4) 393-422.

[2] P. Krishna, N.H. Vaidya, M. Chatterjee, D. Pradhan, (1997) "A cluster-based approach for routing in dynamic networks, ACM SIGCOMM Computer Communication Review“, 27 (2) 49-65.

[3] B. McDonald, T. Znati, (2001) "Design and performance of a distributed dynamic clustering algorithm for Ad-Hoc networks", in: Proceedings of the Annual Simulation Symposium.

[4] V. Mhatre, C. Rosenberg, D. Kofman, R. Mazumdar, N. Shroff, (2004) "Design of surveillance sensor grids with a lifetime constraint", in: 1st European Workshop on Wireless Sensor Networks (EWSN), Berlin.

[5] W.R. Heinzelman, A.P. Chandrakasan, H. Balakrishnan, (2000) "Energy efficient communication protocol for wireless micro sensor networks", in: Proceedings of the 33rd Hawaii International Conference on System Sciences(HICSS-33).

[6] W.R. Heinzelman, A.P. Chandrakasan, H. Balakrishnan, (2002) "An application specific protocol architecture for wireless microsensor networks", IEEE Transactions on Wireless Communications 1 (4) 660-670.

[7] S. Lindsey, C.S. Raghavenda, (2002) PEGASIS: "power efficient gathering in sensor information systems", in: Proceeding of the IEEE Aerospace Conference, Big Sky, Montana.
[8] O. Younis, S. Fahmy, HEED: (2004) "A hybrid, energyefficient, distributed clustering approach for ad hoc sensor networks", IEEE Transactions on Mobile Computing 3, 660-669.

[9] G. Smaragdakis, I. Matta, A. Bestavros, (2004) "SEP: A Stable Election Protocol for clustered heterogeneous wireless sensor network", in: Second International Workshop on Sensor and Actor Network Protocols and Applications (SANPA 2004).

[10] L. Qing, Q. Zhu, M. Wang, (2006) "Design of a distributed energy-efficient clustering algorithm for heterogeneous wireless sensor network", ELSEVIER, Computer Communications 29, pp 2230- 2237.

[11] Elbhiri, B., Saadane, R., El Fkihi, S.,Aboutajdine, D. (2010) "Developed Distributed Energy-Efficient Clustering (DDEEC) for heterogeneous wireless sensor networks", in: 5th International Symposium on I/V Communications and Mobile Network (ISVC).

[12] Parul Saini, Ajay. K. Sharma, (2010) "E-DEECEnhanced Distributed Energy Efficient Clustering Scheme for heterogeneous WSN", $1^{\text {st International }}$ Conference on Parallel, Distributed and Grid Computing (PDGC).

[13] Parul Saini, Ajay.K.Sharma, (2010) "Energy Efficient Scheme for Clustering Protocol Prolonging the Lifetime of Heterogeneous Wireless Sensor Networks", International Journal of Computer Applications (0975 8887), September.

[14] M. Y. Khan, N. Javaid, M. A. Khan, (2013) "Hybrid DEEC: Towards Efficient Energy Utilization in Wireless Sensor Networks", Published in World Applied Science Journal (ISI Indexed).

[15] N. Javaid, T. N. Qureshi, A. H. Khan, A. Iqbal, E. Akhtar, M. Ishfaq, (2013) "EDDEEC: Enhanced Developed Distributed Energy-Efficient Clustering for Heterogeneous Wireless Sensor Networks", ELSEVIER 4th International Conference on Ambient Systems, Networks \& Technologies (ANT).

[16] A. A. Khan, N. Javaid, U. Qasim, Z. Lu, Z. A. Khan, (2012) "HSEP: Heterogeneity-aware Hierarchical Stable Election Protocol for WSNs", IEEE Seventh International Conference on Broadband, Wireless Computing, Communication and Applications.

[17] M. M. Islam, M. A. Matin, T. K. Mondol, (2012) "Extended Stable Election Protocol (ESEP) for Threelevel Hierarchical Clustered Heterogeneous WSN", IEEE Conference on Wireless Sensor Systems (WSS). 